\title{
The Potential of SSR Markers to Reveal the Genetic Diversity among Wheat and its Wild Relatives and to Test the Hybridity of $F_{1} S$
}

\author{
Payal Saxena*, Usha Pant and V. K. Khanna \\ Department of Genetics and Plant Breeding, College of Agriculture, G. B. Pant University of \\ Agriculture and Technology, Pantnagar-263145, Uttarakhand, India \\ *Corresponding author
}

\section{A B S T R A C T}

\section{Keywords}

Genetic diversity, Wheat

(Triticum aestivum), Primers

\section{Article Info}

Accepted:

23 April 2020

Available Online:

10 May 2020
The SSR technique was used to reveal the genetic diversity among wheat (Triticum aestivum) and its wild relatives and secondly to test the hybridity of wheat (Triticum aestivum) $\mathrm{F}_{1} \mathrm{~s}$. All the thirteen SSR primers showed $100 \%$ polymorphism and except one, all the primers generated unique bands, so these primers can be used for genotype identification. Also four primers showed heterozygous nature of $F_{1} s$ by giving two bands, one from each parent at a particular locus, so these markers can be used for screening purpose also. Use of these primers resulted in 180 polymorphic bands out of which 47 were unique.

\section{Introduction}

Bread wheat (Triticum aestivum) is one of the big three globally important crops accounting for $20 \%$ of the calories consumed by the people and a staple crop of nearly $35 \%$ of the global population. The huge bread wheat genome is comprised of $17 \mathrm{~Gb}$ $(17,000,000,000)$ base pairs which is about 5 times the human DNA content and about 40 times of rice genome size. However 80- $90 \%$ of the genome is made up of repetitive sequences. This offers an ample scope for the use of SSRs- the Simple Sequence Repeats as molecular markers studies. Molecular genetics, or the use of molecular techniques for detecting differences in the DNA of individual plants, has got numerous applications in crop improvement.

The differences are called molecular markers because they are often associated with specific genes and act as "signposts" to those genes. Such markers, when very tightly linked to genes of interest, can be used to select indirectly for the desirable allele, and this 
represents the simplest form of Marker Assisted Selection (MAS). Molecular markers used to probe the level of genetic diversity among different cultivars, within populations, among related species, etc. have many applications like varietal fingerprinting for identification and protection, understanding relationships among the units under study, efficiently managing genetic resources, facilitating introgression of chromosomal segments from alien species and even tagging of specific genes (Hoisington et al., 2002).

SSRs involve the use of specifically chosen primers to amplify the repetitive sequences through Polymerase chain reaction. The repetitive DNA of all the species is highly polymorphic in nature. These regions contain genetic loci comprising several hundred alleles, differing from each other with respect to length, sequence or both and they are interspersed in tandem arrays ubiquitously. The term microsatellite was coined by (Litt and Lutty 1989). SSRs are increasingly being used as genetic markers of chromosome segments (Dib et al., 1996), for identification of individuals (Anon, 1996), studying evolution and orthologous and paralogous relatedness (Rubinsztein et al., 1995 and Ali et al., 1999) and wildlife conservation (Roca et al., 2001). The present study aimed at the use of SSRs to study the genetic diversity of wheat and its wild relatives at all ploidy levels (diploid, tetraploid and hexaploid) and secondly the codominant marker was also used to to test the hybridity of wheat ( $T$. aestivum) $\mathrm{F}_{1} \mathrm{~s}$.

\section{Materials and Methods}

The study was conducted at G.B. Pant University of Agriculture \& Technology, Pantnagar, Uttarakhand, India during 200408 . The seeds of wild relatives of wheat were obtained from Directorate of Wheat Research, Karnal, Haryana, India. The experimental material consisted of 41 genotypes which included 10 wild relatives of wheat, 2 Triticum durum varieties, 15 Triticum aestivum varieties and $14 \mathrm{~F}_{1} \mathrm{~s}$ among them. The parentage of $T$. aestivum genotypes is given in Table 1. DNA characterization was done using 13 SSR primers. Primers were provided by Integrated DNA Technologies, Inc. Details of primers are given in Table 2.

\section{Genomic DNA extraction}

CTAB procedure was used for the isolation of DNA. CTAB (Cetyl trimethyl ammonium bromide) is a cationic detergent which solubilizes membranes and forms a complex with DNA. After cell disruption and incubation with hot CTAB isolation buffer, proteins were extracted by chloroform: isoamyl alcohol. CTAB-DNA was precipitated with isopropanol. The DNA pellet resulting after centrifugation was washed, dried and redissolved. RNase A treatment was given to remove RNA contamination.

\section{Protocol followed}

Two $g$ of fresh wheat seedling leaves were ground to a fine powder using liquid nitrogen and a mortar and pestle.

The powder was transferred as fast as possible into $15 \mathrm{ml}$ of pre-warmed $\left(60^{\circ} \mathrm{C}\right)$ isolation buffer in an oakridge tube.

The oakridge tubes were then incubated in a water bath at $60^{\circ} \mathrm{C}$ for 30 minutes. It was mixed gently after every 10 minutes.

One volume of chloroform: isoamyl alcohol (24: 1) was then added. The tube was capped and shaken gently and thoroughly for 10 minutes by hand, enough to ensure emulsification of the phase.

Then it was centrifuged for 10 minutes (5000 rpm, room temperature). The (upper) aqueous phase was extracted once again with fresh chloroform: isoamyl alcohol.

The final aqueous phase was transferred to a 
fresh tube using micropipette with a wide bore microtip (that of $1000 \mu$ l capacity).

0.6 volume of chilled isopropanol was added, the tube was capped and mixing was done gently but thoroughly by inverting the tube several times. At this stage, the DNA-CTAB complex precipitated as a whitish network. The solution was placed at $-20^{\circ} \mathrm{C}$ for 30 minutes to overnight.

Then it was centrifuged (10 min., $5000 \mathrm{rpm}$, $\left.4^{\circ} \mathrm{C}\right)$. It was then washed with $70 \%$ ethanol; the pellet was gently agitated for a few minutes, and collected by centrifugation $\left(10 \mathrm{~min} ., 5000 \mathrm{rpm}, 4^{\circ} \mathrm{C}\right)$. Residual CTAB was removed by this step. The tubes were inverted and drained on a paper towel for about 1 hour taking care that pellet does not slip down the wall of the tube. It was ensured that it neither contained residual ethanol nor it was too dry. In both cases redissolving might be difficult.

An appropriate volume of $1 \mathrm{X}$ TE buffer was added (say $500 \mu \mathrm{l}$ ) and the pellet was allowed to dissolve at $4^{\circ} \mathrm{C}$ without agitation.

\section{Purification and quantification of genomic DNA}

$5 \mu \mathrm{R}$ Rase $(10 \mathrm{mg} / \mathrm{ml})$ was added to $100 \mu \mathrm{l}$ of dissolved DNA and incubated at $37^{\circ} \mathrm{C}$ for 1 hour. Equal volume of phenol: chloroform: isoamyl alcohol (25: 24: 1) was added and mixed gently by inverting the tubes. The tubes were spun at $10,000 \mathrm{rpm}$ for 5 minutes and aqueous layer (i.e. upper layer) was collected and equal amount of chloroform + isoamyl alcohol (24: 1) was added. The tubes were spun at 10,000 rpm for $5 \mathrm{~min}$ and the top layer of DNA was removed. To this, sodium acetate $(1 / 10$ vol, $\mathrm{pH}=5.2)$ and chilled absolute ethanol was added. The contents were mixed and kept at $-20^{\circ} \mathrm{C}$ for $30 \mathrm{~min}$. Finally the pellet was washed with 70 per cent ethanol, dried and dissolved in $100 \mu \mathrm{TE}$ buffer.

The quantification of genomic DNA was done by taking the absorbance on Genesys UV spectrophotometer. The optical density was measured at 260 and $280 \mathrm{~nm}$. The concentration of the DNA in the sample is related to optical density by the following formula:

Conc. of DNA $(\mu \mathrm{g} / \mathrm{ml})=\frac{\mathrm{OD}_{260} \times 50 \times \text { Dilution factor }}{1000}$

The ratio of $\mathrm{OD}_{260 / 280}$ was an indication of the amount of RNA or protein contamination in the preparation. A value of 1.8 is optimum for the best DNA preparation. A value of the ratio below 1.8 indicates the presence of protein in the preparation and a value above 1.8 indicates that the sample has RNA contamination.

\section{PCR amplification}

The reaction mixture consisted of genomic DNA, d NTPs, Taq polymerase, reaction buffer, primers (forward and reverse) and double distilled water. The concentrations and quantity of components is given in Table 3. The PCR thermocycler was programmed according to the Table 4. In PCR programming 3 annealing temperatures were used: $51^{\circ} \mathrm{C}$ for Barc 019, Barc 119, Barc 025, Barc 028, Barc 062, Barc 065, Barc 142, Barc 154 and Barc 228. $52^{\circ} \mathrm{C}$ for Barc 003 and Barc 111 and $54^{\circ} \mathrm{C}$ for Barc 124 and Barc 159.

Electrophoresis of the amplified PCR products was done in horizontal gel electrophoresis assembly using agarose gel of $2.5 \%$ concentration. Electrophoresis was done at $50 \mathrm{~V}$ for 4 hours in $0.5 \mathrm{X}$ TBE buffer. After $75 \%$ run of the gel, its image was viewed and its photograph saved in a gel documentation system. 


\section{Data analysis}

Gels were documented using Gel Doc system (Bio-Rad). Pair-wise similarity and cluster analysis were done on the basis of presence and absence of bands. Computer software (NTSYS) was used to perform the similarity matrix analysis using 'UPGMA' with Jaccard's coefficient of similarity.

\section{Results and Discussion}

All the13 SSR primers used in the study were polymorphic. They amplified total 180 bands out of which 47 bands were unique. 12 primers gave unique bands. The size of bands ranged from 100 to $3000 \mathrm{bp}$. The details of amplification pattern are provided in the Table 5.

All the thirteen SSR primers showed 100\% polymorphism and except one, all the primers generated unique bands, so these primers can be used for genotype identification. Also four primers showed heterozygous nature of F1s by giving two bands, one from each parent at a particular locus, so these markers can be used for screening purpose also. Primer Barc 019 amplified maximum number of loci (24) and also gave maximum number of unique bands (10) followed by Barc 062 (7), Barc 142 (6), Barc 028 (5), and Barc 228 (4), whereas Barc 065, Barc 119 and Barc 154, each gave 3 unique bands whereas Barc 145, Barc 159 and Barc 025 gave 2, Barc 124 gave one unique band. So these markers can be used for the identification of genotypes.

The wild species $T$. dicoccum showed highest number of unique bands (18) from 6 primers - Barc 019, Barc 025, Barc 142, Barc 154, Barc 159 and Barc 228 followed by Ae. Squarrosa (5) from 4 primers - Barc 019, B. Other wild species $T$. sphaerococcum, $T$. polonicum, T. monococcum also showed unique bands. T. durum variety PDW 289 and Secale cereale accession EC 481695 also showed unique bands. It can be inferred that these wild germplasms harbour drought tolerance characteristics and can be used as donor of drought tolerance trait in wheat breeding programmes.

T. aestivum variety WH 730 showed maximum number of unique bands. Varieties like UP 2565 and PBW 373 also showed unique bands which incates the possibility of developing drought tolerance in these varieties. UP 2425 showed 4 unique bands. The study of R. P. Meena et al., (2015) also suggests that UP 2425 performs better under moisture stress conditions based on several stress indices. The hills variety VL 804 also showed unique band confirming its drought tolerant nature. The cross Job 666 X UP 2565 showed unique band as well as the bands present at a particular locus in the parents were also present in the cross, primer Barc 154. This codominant nature of marker was shown in the cross NP 846 x UP 2425 by 2 primers Barc 028 and Barc 159. Barc 025 also revealed its codominant nature in the cross NIAW 34 x UP 2590 (Fig. 1-3).

\section{SSR cluster analysis}

The dendrogram that was constructed using NTSYS software divided the genotypes in several clusters. Firstly 2 major groups were formed. Group 1 comprised of genotypes 1,2,11 -Secale cereale EC 481697, Secale cereale EC 481695 and T. dicoccum. All other genotypes formed group 2. Group 2 was further divided in group 2a and group $2 \mathrm{~b}$.

$2 \mathrm{a}$ comprised of $2 \mathrm{a}$ sub group 1 and $2 \mathrm{a}$ sub group 2. First cluster of $2 \mathrm{a}$ sub group 1 included the genotypes 3,12, 15, 13, 5, 6, 7 T. timopheevii, WH730 x UP 2425, Job 666 x UP 2565, Job 666 x UP 2425, T. tauschii, T. sphaerococcum, Ae. Squarrosa. Out of these T. timopheevii was clustered as a separate small cluster, WH 730 x UP 2425, Job 666 x UP 2565 and Job 666 x UP 2425 in another 
small cluster. T. tauschii, T. sphaerococcum and Ae. squarrosa in a third small cluster. Second cluster of $2 \mathrm{a}$ sub group 1 had genotypes 14 and 16 - Job 666 and UP 2565.

2a sub group 2 had $4,8,9,10-T$. polonicum, T. turgidum, PDW 291 and PDW 289. Out of these $T$. polonicum existed as a separate cluster other than T. turgidum, PDW 291 and PDW 289. Group $2 b$ had 2 sub-groups $2 b$ sub group 1 and $2 b$ sub group 2 . $2 b$ sub group 1 existed as a single genotype $17-\mathrm{T}$. monococcum.

Rest other genotypes were present in $2 \mathrm{~b}$ sub group 2 i.e. $18,19,23,24,25,27,28,31,29$, $33,34,20,21,22,30,32,35,40,41,38,39$, 26, 36, 37 - Halna, PBW 175, PBN 51 x UP 2554, UP 2554, WH 730 x UP 2554, WH730 $x$ UP 2338, UP 2338, NIAW 34 x PBW 373, PBN $51 \times$ UP 2338, NIAW x UP 2590, UP 2590, VL 804, PBN 51 x VL 804, PBN 51, PBW 373, NIAW 34, NIAW 34 x UP 2565, HI 385 x UP 2425, HI 385, NP 846 x UP 2425, NP 846, WH 730, NIAW 34 x UP 2425, UP 2425.

\section{Relationship among wheat genotypes}

Based on the estimated genetic similarity matrix using UPGMA method, the primers revealed highest genetic similarity value 0.7895 between VL 804 and its cross PBN 51 $x$ VL 804 indicating the involvement of drought lines, followed by 0.7143 between 2 crosses WH 730 x UP 2425 and Job 666 X UP 2565 indicating the presence of drought tolerant parents WH 730 \& Job 666 in the crosses, also the other parents UP 2425 and UP 2565 are the varieties released from the same place i.e. Pantnagar and both are recommended for irrigated late sown conditions. It was followed by the similarity value 0.6857 between $\mathrm{HI} 385$ and its cross $\mathrm{HI}$ 385 x UP 2425, followed by 0.6786 between PBW 175 and a cross PBN 51 x UP2554 as PBW 175 is drought tolerant and the parental line PBN 51 of the cross is also drought tolerant. It was followed by the similarity value 0.6765 between UP 2590 and its cross NIAW 34 x UP 2590, followed by 0.6744 between 2 crosses Job 666 X UP 2425 and Job $666 \times$ UP 2565 due to the common parent Job 666 between them.

It was followed by the similarity value 0.6667 between 2 Triticum durum varieties PDW 291 and PDW 289, followed by 0.6563 between UP 2338 and a cross NIAW 34 x PBW 373. It was followed by the similarity value 0.6486 between UP 2554 and its cross WH 730 x UP 2554 , followed by 0.6250 in 3 pairs i.e. between UP 2338 and its cross PBN 51 x UP 2338, between 2 crosses PBN 51 x UP 2554 and PBN 51 x UP 2338 and between Halna and PBW 175.

The results indicate that SSRs can be very effectively used for molecular characterization of genotypes. SSRs are codominant markers which show bands in both the parents at different loci as well as both the parental bands in the cross. As we know that drought tolerance is a complex Quantitative trait loci therefore a lot of variations can be observed in the banding pattern of crosses. Further for QTL mapping or Gene tagging purposes the populations like Nearly Isogenic Lines, Doubled Haploids, Recombinant Inbred Lines, $F_{2}$, Back cross should be used. 
Table.1 List of various wheat (Triticum aestivum, genome AABBDD, 2n= 42)

\begin{tabular}{|c|c|c|}
\hline Varieties & Parentage & Remarks \\
\hline Halna (K 7903) & HD 1982 / K 816 & Drought tolerant (gene introgressed) \\
\hline UP 2565 & PBW 352 / CPAN 4020 & \\
\hline HI 385 (MUKTA) & HYB 633 // GAZA // PR / PKD 25 & Drought tolerant (gene introgressed) \\
\hline PBW 373 & $\begin{array}{l}\text { ND / VG } 9144 \text { // KAL / BB / } 3 \text { / YACO '5' / } \\
4 \text { / VEE \# } 5 \text { 'S' }\end{array}$ & \\
\hline NIAW 34 & CNO 79 / PRL “S” & Drought tolerant (gene introgressed) \\
\hline UP 2425 & HD 2320/UP 2263 & Drought susceptible \\
\hline NP 846 & NP 760 / RN & Drought tolerant (gene introgressed) \\
\hline UP 2338 & UP 368 / VL 421 / UP 262 & Drought susceptible \\
\hline PBW 175 & HD 2160 / WG 1025 & Drought tolerant (gene introgressed) \\
\hline PBN 51 & BUC 'S’ / FLK 'S’ & Drought tolerant (gene introgressed) \\
\hline UP 2554 & SM4 - HSN 24E / CPAN 2099 & \\
\hline UP 2590 & Not available & \\
\hline VL 804 & CPAN 3018/CPAN 3004//PBW 65 & Drought tolerant \\
\hline WH 730 & CPAN 2092/ Improved Lok- 1 & $\begin{array}{l}\text { Drought tolerant } \\
\text { (gene introgressed) }\end{array}$ \\
\hline JOB 666 & K 65 / HD 2009 & Drought tolerant (gene introgressed) \\
\hline
\end{tabular}

List of related species

\begin{tabular}{|c|c|c|c|c|}
\hline Species & $\begin{array}{c}\text { Variety/ } \\
\text { accessions }\end{array}$ & Parentage & Genome & $\begin{array}{l}\text { Chromosome } \\
\text { no. }\end{array}$ \\
\hline Aegilops squarrosa & - & - & $\mathrm{DD}$ & 14 \\
\hline $\begin{array}{c}\text { Triticum } \\
\text { monococcum }\end{array}$ & - & - & AA & 14 \\
\hline T. tauschii & - & - & DD & 14 \\
\hline T. dicoccum & - & - & $\mathrm{AABB}$ & 28 \\
\hline \multirow[b]{2}{*}{ T. durum } & PDW 289 & - & $\mathrm{AABB}$ & 28 \\
\hline & PDW 291 & $\begin{array}{c}\text { BOOMER 21/ } \\
\text { MOJO } 2\end{array}$ & AABB & 28 \\
\hline T. turgidum & - & - & $\mathrm{AABB}$ & 28 \\
\hline T. polonicum & - & - & $\mathrm{AABB}$ & 28 \\
\hline T. sphaerococcum & - & - & AABBDD & 42 \\
\hline T. timopheevii & - & - & AAGG & 28 \\
\hline Secale cereale & EC 481695 & - & RR & 14 \\
\hline Secale cereale & EC 481697 & - & RR & 14 \\
\hline
\end{tabular}


Table.2 Characteristics of SSR Primers

\begin{tabular}{|c|c|c|c|c|c|}
\hline $\begin{array}{l}\text { SI. } \\
\text { No. }\end{array}$ & Operon Code & $\begin{array}{c}\text { Forward } \\
\text { Sequence }\left(5^{\prime}-3^{\prime}\right)\end{array}$ & $\begin{array}{c}\text { GCcontent } \\
(\%)\end{array}$ & $\begin{array}{c}\text { Reverse } \\
\text { Sequence(5'-3') }\end{array}$ & GC content $(\%)$ \\
\hline 1. & Barc 003 & $\begin{array}{l}\text { TTCCCTGTGTCTT } \\
\text { TCTAATTTTTTTT }\end{array}$ & 26.9 & $\begin{array}{l}\text { GCGAACTCCCG } \\
\text { AACATTTTTAT }\end{array}$ & 40.9 \\
\hline 2. & Barc 019 & $\begin{array}{l}\text { GCGACCCGAGTA } \\
\text { GCCTGAA }\end{array}$ & 63.1 & $\begin{array}{l}\text { GGTGGACCATTA } \\
\text { GACGCTTACTTG }\end{array}$ & 50.0 \\
\hline 3. & Barc 025 & $\begin{array}{l}\text { GCGGTGCATCAA } \\
\text { GGACGACAT }\end{array}$ & 57.1 & $\begin{array}{l}\text { GCGTAGTTC } \\
\text { ATCCACCGTAAT }\end{array}$ & 45.4 \\
\hline 4. & Barc 028 & $\begin{array}{l}\text { CTCCCCGGCTAG } \\
\text { TGACCACA }\end{array}$ & 65.0 & $\begin{array}{l}\text { GCGGCATCTTTCA } \\
\text { TTAACGAGCTAGT }\end{array}$ & 46.1 \\
\hline 5. & Barc 062 & $\begin{array}{l}\text { TTGCCTGAGACAT } \\
\text { ACATACACCTAA }\end{array}$ & 40.0 & $\begin{array}{l}\text { GCCAGAACAGAA } \\
\text { TGAGTGCT }\end{array}$ & 50.0 \\
\hline 6. & Barc 065 & $\begin{array}{l}\text { CCCATGGCCAAG } \\
\text { TATAATAT }\end{array}$ & 40.0 & $\begin{array}{l}\text { GCGAAAAGTCCAT } \\
\text { AGTCCATAGTCTC }\end{array}$ & 46.1 \\
\hline 7. & Barc 119 & $\begin{array}{l}\text { CACCCGATGATGA } \\
\text { AAAT }\end{array}$ & 41.1 & $\begin{array}{l}\text { GATGGCACAAG } \\
\text { AAATGAT }\end{array}$ & 38.8 \\
\hline 8. & Barc 124 & $\begin{array}{l}\text { TGCACCCCTTCC } \\
\text { AAATCT }\end{array}$ & 50.0 & $\begin{array}{l}\text { TGCGAGTCGTGT } \\
\text { GGTTGT }\end{array}$ & 55.5 \\
\hline 9. & Barc 142 & $\begin{array}{l}\text { CCGGTGAGAGGA } \\
\text { CTAAAA }\end{array}$ & 50.0 & $\begin{array}{l}\text { GGCCTGTCAATT } \\
\text { ATGAGC }\end{array}$ & 50.0 \\
\hline 10. & Barc 145 & $\begin{array}{l}\text { GCAGCCTCGA } \\
\text { ATCACA }\end{array}$ & 56.2 & $\begin{array}{l}\text { GGGGTGTTGAAG } \\
\text { ATGA }\end{array}$ & 50.0 \\
\hline 11. & Barc 154 & $\begin{array}{l}\text { GTAATTCCGGTT } \\
\text { CCACTTGACATT }\end{array}$ & 55.5 & $\begin{array}{l}\text { GGATGGGCAGCT } \\
\text { TCAAGGTATGTT }\end{array}$ & 50.0 \\
\hline 12. & Barc 159 & $\begin{array}{l}\text { CGCAATTTATTAT } \\
\text { CGGTTTTAGGAA }\end{array}$ & 32.0 & $\begin{array}{l}\text { CGCCCGATAGTTT } \\
\text { TTCTAATTTCTGA }\end{array}$ & 38.4 \\
\hline 13. & Barc 228 & $\begin{array}{l}\text { CCСТCСТCTCT } \\
\text { TTAGCCATCC }\end{array}$ & 57.1 & $\begin{array}{l}\text { GCACGTACTATTC } \\
\text { GCCTTCACTTA }\end{array}$ & 56.8 \\
\hline
\end{tabular}

Table.3 Standard concentration of components for PCR amplification

\begin{tabular}{|l|c|c|}
\hline Components (Conc.) & Final Conc./25 $\boldsymbol{\mu l}$ & Single tube $\boldsymbol{\mu l}$ \\
\hline DNA template $(20 \mathrm{ng} / \mu \mathrm{l})$ & $40 \mathrm{ng}$ & $2.0 \mu \mathrm{l}$ \\
\hline $\mathrm{d}$ NTPs $(2.5 \mathrm{mM}$ each $)$ & $200 \mu \mathrm{M}$ each & $2.0 \mu \mathrm{l}$ \\
\hline Taq polymerase $(3 \mathrm{U} / \mu \mathrm{l})$ & $0.76 \mathrm{U}$ & $0.5 \mu \mathrm{l}$ \\
\hline Reaction buffer $(10 \mathrm{X})$ & $1 \mathrm{X}$ & $2.5 \mu \mathrm{l}$ \\
\hline Primer $(50 \mathrm{ng} / \mu \mathrm{l})$ forward & $50 \mathrm{ng}$ & $1.0 \mu \mathrm{l}$ \\
\hline Primer $(50 \mathrm{ng} / \mu \mathrm{l})$ reverse & $50 \mathrm{ng}$ & $1.0 \mu \mathrm{l}$ \\
\hline $\mathrm{dd} \mathrm{H}_{2} \mathrm{O}$ & & $16.0 \mu \mathrm{l}$ \\
\hline & Total & $\mathbf{2 5 . 0} \mu \mathrm{l}$ \\
\hline
\end{tabular}

Table.4 Protocol for PCR amplification

\begin{tabular}{|l|c|c|c|c|c|c|}
\hline \multirow{2}{*}{ Cycle } & \multicolumn{2}{|c|}{ Denaturation } & \multicolumn{2}{|c|}{ Annealing } & \multicolumn{2}{c|}{ Polymerization } \\
\cline { 2 - 7 } & Temp. & Time & Temp. & Time & Temp. & Time \\
\hline First cycle & $94^{\circ} \mathrm{C}$ & $5 \mathrm{~min}$ & - & - & - & - \\
\hline 44 cycles & $94^{\circ} \mathrm{C}$ & $1 \mathrm{~min}$ & $51^{\circ} \mathrm{C}, 52^{\circ} \mathrm{C}$, & $1 \mathrm{~min}$ & $72^{\circ} \mathrm{C}$ & $2 \mathrm{~min}$ \\
& & & $54^{\circ} \mathrm{C}$ & & & \\
\hline Last cycle & - & - & - & - & $72^{\circ} \mathrm{C}$ & $7 \mathrm{~min}$ \\
\hline
\end{tabular}


Table.5 Details of amplification pattern

\begin{tabular}{|c|c|c|c|c|c|c|c|c|c|}
\hline \multirow{3}{*}{$\begin{array}{c}\text { S.No. } \\
1 .\end{array}$} & \multirow{3}{*}{$\begin{array}{c}\begin{array}{c}\text { Name of } \\
\text { Primer }\end{array} \\
\text { Barc } 003\end{array}$} & \multirow{3}{*}{$\begin{array}{c}\begin{array}{c}\text { No. of } \\
\text { amplified } \\
\text { loci }\end{array} \\
10\end{array}$} & \multirow{3}{*}{$\begin{array}{c}\begin{array}{c}\text { Size of } \\
\text { bands } \\
\text { (bp) }\end{array} \\
150- \\
3000 \\
\end{array}$} & \multirow{3}{*}{$\begin{array}{l}\text { No. of } \\
\text { Unique } \\
\text { bands }\end{array}$} & \multicolumn{2}{|c|}{ Genotypes having unique bands } & \multicolumn{3}{|c|}{ Codominant nature of marker } \\
\hline & & & & & \multirow[t]{2}{*}{ Genotype } & \multirow[t]{2}{*}{ Size of unique band (bp) } & $\begin{array}{l}\text { Bands in } \\
\text { cross }\end{array}$ & \multicolumn{2}{|c|}{ Bands in parents } \\
\hline & & & & & & & & & \\
\hline \multirow[t]{10}{*}{2.} & \multirow[t]{10}{*}{ Barc 019} & \multirow[t]{10}{*}{24} & \multirow{10}{*}{$\begin{array}{l}110- \\
3000\end{array}$} & \multirow[t]{10}{*}{10} & Ae. squarrosa & 3000 & & & \\
\hline & & & & & UP 2425 & 1200 & & & \\
\hline & & & & & T. dicoccum & 1000 & & & \\
\hline & & & & & & 850 & & & \\
\hline & & & & & & 520 & & & \\
\hline & & & & & & 410 & & & \\
\hline & & & & & & 390 & & & \\
\hline & & & & & & 340 & & & \\
\hline & & & & & $\begin{array}{c}\text { NIAW } 34 x \\
\text { UP2425 }\end{array}$ & 330 & & & \\
\hline & & & & & UP 2565 & 200 & & & \\
\hline \multirow[t]{2}{*}{3.} & \multirow[t]{2}{*}{ Barc 025} & \multirow[t]{2}{*}{8} & \multirow{2}{*}{$\begin{array}{l}200- \\
2800\end{array}$} & \multirow[t]{2}{*}{2} & \multirow[t]{2}{*}{ T. dicoccum } & 550 & \multirow{2}{*}{$\begin{array}{c}\text { NIAW } 34 \\
\text { x UP } 2590 \\
250 \mathrm{bp} \& \\
200 \mathrm{bp}\end{array}$} & \multirow{2}{*}{$\begin{array}{c}\text { NIAW3 } \\
4 \\
250 \mathrm{bp}\end{array}$} & \multirow[t]{2}{*}{ UP 2590} \\
\hline & & & & & & 360 & & & \\
\hline \multirow[t]{5}{*}{4.} & \multirow[t]{5}{*}{ Barc 028} & \multirow[t]{2}{*}{19} & \multirow{2}{*}{$\begin{array}{l}230- \\
2000\end{array}$} & \multirow[t]{2}{*}{5} & \multirow[t]{2}{*}{ Ae. squarrosa } & 2000 & \multirow{5}{*}{$\begin{array}{l}\text { NP } 846 x \\
\text { UP } 2425 \\
270 \mathrm{bp} \\
250 \mathrm{bp}\end{array}$} & & UP 2425 \\
\hline & & & & & & 1500 & & 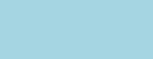 & \\
\hline & & & & & Job 666 x UP 2565 & 750 & & $270 \mathrm{bp}$ & \\
\hline & & & & & T. sphaerococcum & 300 & & & $250 \mathrm{bp}$ \\
\hline & & & & & T. polonicum & 260 & & & \\
\hline 5. & Barc 062 & 13 & $\begin{array}{l}100- \\
1200\end{array}$ & 7 & WH 730 & 1200 & & & \\
\hline & & & & & & 900 & & & \\
\hline & & & & & & 700 & & & \\
\hline & & & & & & 600 & & & \\
\hline
\end{tabular}




\begin{tabular}{|c|c|c|c|c|c|c|c|c|c|}
\hline & & & & & & 520 & & & \\
\hline & & & & & & 375 & & & \\
\hline & & & & & & 360 & & & \\
\hline \multirow[t]{3}{*}{6.} & \multirow[t]{3}{*}{ Barc 065} & \multirow[t]{3}{*}{7} & \multirow{3}{*}{$\begin{array}{c}100- \\
490\end{array}$} & \multirow[t]{3}{*}{3} & \multirow[t]{2}{*}{ T. monocoсcum } & 410 & & & \\
\hline & & & & & & 290 & & & \\
\hline & & & & & PBW 373 & 275 & & & \\
\hline \multirow[t]{3}{*}{7.} & \multirow[t]{3}{*}{ Barc 119} & \multirow[t]{3}{*}{20} & \multirow{3}{*}{$\begin{array}{l}180- \\
1400\end{array}$} & \multirow[t]{3}{*}{3} & \multirow[t]{3}{*}{ UP 2425} & 580 & & & \\
\hline & & & & & & 330 & & & \\
\hline & & & & & & 310 & & & \\
\hline 8. & Barc 124 & 12 & $\begin{array}{l}210- \\
1100\end{array}$ & 1 & HI 385 & 1000 & & & \\
\hline \multirow[t]{6}{*}{9.} & \multirow[t]{6}{*}{ Barc 142} & \multirow[t]{6}{*}{18} & \multirow{6}{*}{$\begin{array}{l}160- \\
1500\end{array}$} & \multirow[t]{6}{*}{6} & \multirow[t]{4}{*}{ T. dicoccum } & 1500 & & & \\
\hline & & & & & & 720 & & & \\
\hline & & & & & & 480 & & & \\
\hline & & & & & & 250 & & & \\
\hline & & & & & PDW 289 & 200 & & & \\
\hline & & & & & $\begin{array}{c}\text { S. cereale EC } \\
481695\end{array}$ & 800 & & & \\
\hline \multirow[t]{2}{*}{10.} & \multirow[t]{2}{*}{ Barc 145} & \multirow[t]{2}{*}{17} & \multirow{2}{*}{$\begin{array}{l}150- \\
1200\end{array}$} & \multirow[t]{2}{*}{2} & Ae. squarrosa & 440 & & & \\
\hline & & & & & PBW 373 & 320 & & & \\
\hline \multirow[t]{3}{*}{11.} & \multirow[t]{3}{*}{ Barc 154} & \multirow[t]{3}{*}{10} & \multirow{3}{*}{$\begin{array}{c}250- \\
600\end{array}$} & \multirow[t]{3}{*}{3} & T. dicoccum & 500 & \multirow{3}{*}{$\begin{array}{c}\text { Job } 666 \text { x } \\
\text { UP } 2565 \\
300 \text { bp } \\
280 \text { bp }\end{array}$} & \multirow[t]{3}{*}{ Job 666} & UP 2565 \\
\hline & & & & & Ae. squarrosa & 480 & & & \\
\hline & & & & & T. polonicum & 320 & & & $300 \mathrm{bp}$ \\
\hline 12. & Barc 159 & 8 & $180-$ & 2 & T. dicoccum & 700 & NP $846 x$ & NP 846 & UP 2425 \\
\hline & & & 700 & & & 400 & UP 2425 & & \\
\hline & & & & & & & $\begin{array}{l}220 \mathrm{bp} \\
210 \mathrm{bp}\end{array}$ & $210 \mathrm{bp}$ & 220 bp \\
\hline 13. & Barc 228 & 14 & $180-$ & 4 & T. dicoccum & 1500 & & & \\
\hline & & & 1500 & & & 520 & & & \\
\hline & & & & & & 270 & & & \\
\hline & & & & & VL 804 & 230 & & & \\
\hline
\end{tabular}



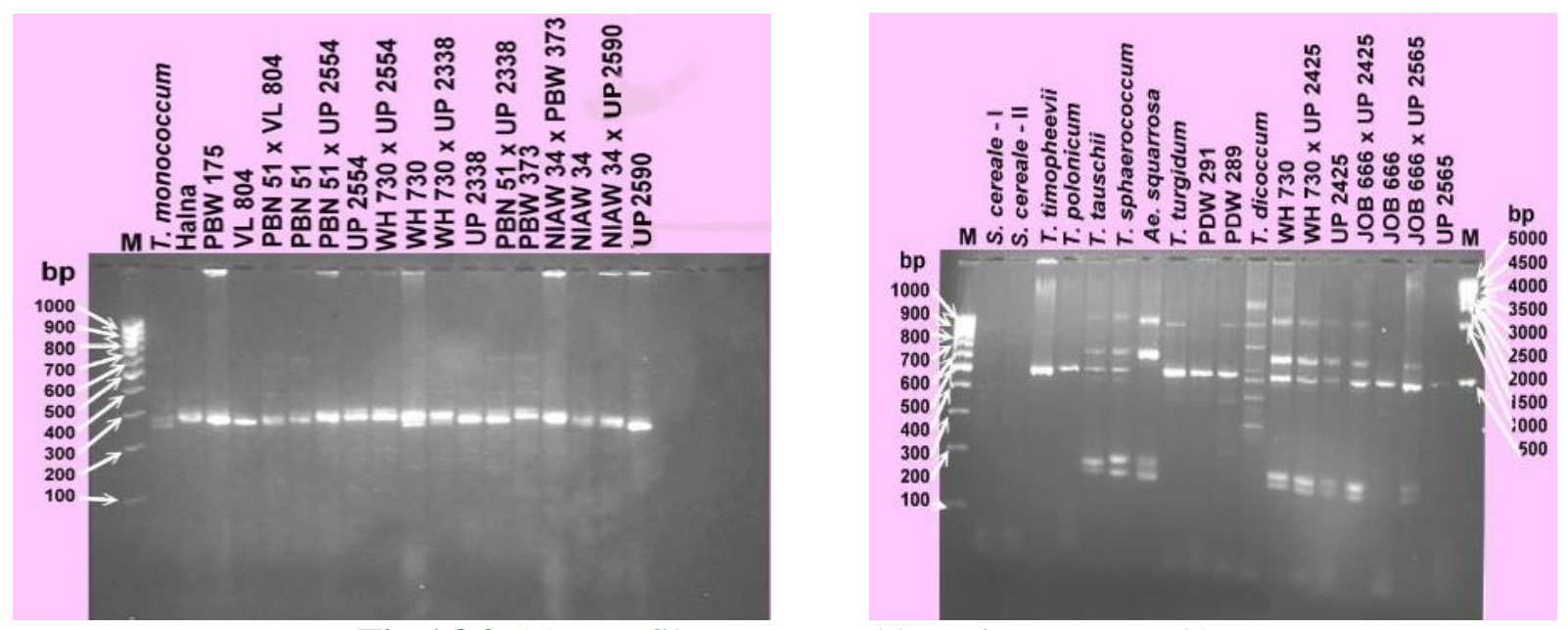

Fig.1\&2 SSR profiles generated by primer Barc 142

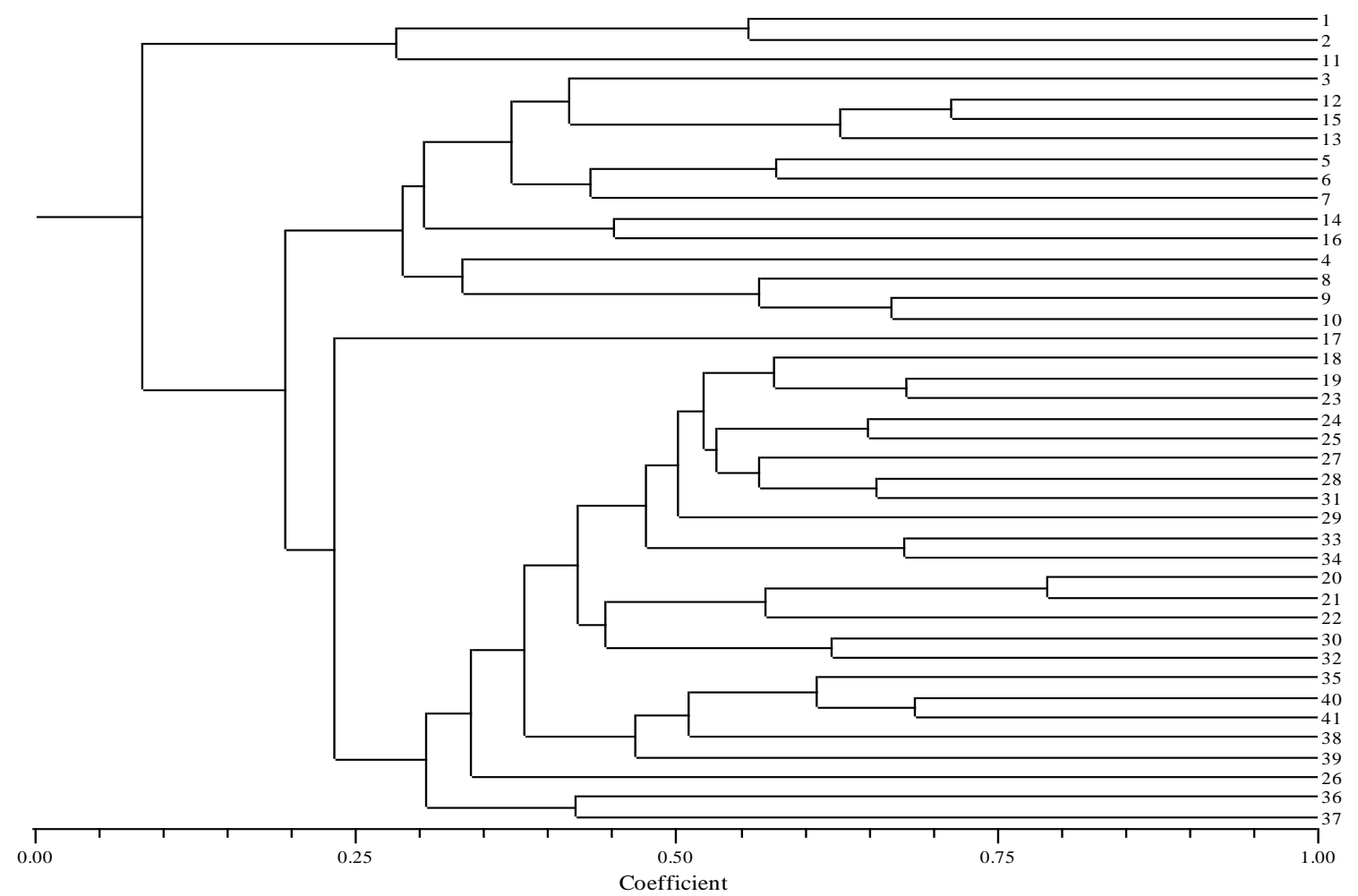

Fig.3 Dendrogram of wheat genotypes constructed using Jaccard's coefficient of similarity

Wheat genotypes as represented in SSR dendrogram
1. Secale cereale EC 481697
2.Secale cereale EC 481695
3. Triticum timopheevii
4. Triticum polonicum
5. Triticum tauschii
6.Triticum sphaerococcum
7.Aegilops squarrosa
8.Triticum turgidum
9. PDW 291
10. PDW 289
11. Triticum dicoccum
12.WH 730 x UP 2425
13. JOB 666 x UP 2425
14.JOB 666
15.JOB 666 x UP 2565
16. UP 2565
17.Triticum monococcum
18. HALNA
19. PBW 17
20.VL 804
21. PBN $51 \times$ VL 804
22. PBN 51
23. PBN 51 x UP 2554
25. WH 730 x UP 2554 26. WH 730
27. WH 730 x UP 2338
28. UP 2338
29. PBN 51 x UP 2338 30. PBW 373
31. NIAW 34 x PBW 373 32. NIAW 34
33. NIAW 34 x HD 2590
34. HD 2590
35. NIAW 34 x UP 2565
36.NIAW 34 x UP 2425
37. UP 2425
38. NP 846 x UP 2425
39.NP 846
40.HI 385 x UP 2425
41.HI 385 
The SSR primers revealed the lowest genetic similarity value 0.0208 between Secale cereale EC 481697 and Triticum monococcum, followed by 0.0282 between Triticum dicoccum and Triticum monococcum, followed by 0.0290 between Triticum dicoccum and a cross NIAW $34 \mathrm{x}$ UP 2590, followed by 0.0364 between Secale cereale EC 481695 and VL 804, followed by 0.0380 between Aegilops squarrosa and Triticum dicoccum, followed by 0.0385 between Secale cereale EC 481695 and UP 2554, followed by 0.0390 between Secale cereale EC 481697 and UP 2425, followed by 0.0392 between Secale cereale EC 481695 and Triticum monococcum, followed by 0.0400 between Triticum dicoccum and NIAW 34, followed by 0.0408 between Secale cereale EC 481695 and a cross NIAW 34 x PBW 373, followed by 0.0426 between Secale cereale EC 481697 and Halna, followed by 0.0435 between Triticum dicoccum and UP 2590, followed by 0.0444 between Secale cereale EC 481697 and a cross NIAW 34 x UP 2590, followed by 0.0448 between Triticum dicoccum and a cross PBN 51 x UP 2338, followed by 0.0455 between 2 pairs i.e. between Secale cereale EC 481697 and a cross PBN 51 x UP 2338 and between Triticum teemopheevii and Triticum dicoccum, followed by 0.0462 between 2 pairs i.e. between Triticum dicoccum and UP 2338 and between Triticum dicoccum and a cross PBN 51 x UP 2554, followed by 0.0469 between Triticum dicoccum and PBN 51, followed by 0.0476 between Secale cereale EC 481697 and UP 2338, followed by 0.0484 between Triticum dicoccum and NP 846, followed by 0.0488 between Secale cereale EC 481697 and PBN 51 and so on.

The low similarity matrix values clearly indicate low genetic similarities or high genetic diversity and may be attributed to genomic differences in the study material.
Relationship among wheat and its wild relatives

SSR amplification patterns were analyzed to study the genetic diversity between wheat and its wild relatives. The $\mathrm{F}_{1} \mathrm{~s}$ were excluded from this analysis. Based on the estimated genetic similarity the highest genetic similarity value (0.667) was observed between PDW 291 and PDW 289, as both of them are the two varieties of $T$. durum followed by 0.625 between Halna and PBW 175, followed by 0.620 between PBW 373 and NIAW 34, followed by 0.604 between UP 2554 and PBW 373, followed by 0.578 between $T$. tauschii and T. sphaerococcum, followed by 0.556 between Secale cereale EC 481697 and Secale cereale EC 48195 , followed by 0.550 between T. turgidum and PDW 291 followed by 0.545 between PBW 175 and UP2590, followed by 0.525 between Halna and UP 2554, followed by 0.516 between PBW 175 and UP 2338 followed by 0.514 between 2 pairs i.e. UP2554 and UP 2338; PBW 175 and UP 2554.

The lowest genetic similarity value 0.021 was observed between Secale cereal EC 481697 and $T$. monococcum, followed by 0.028 between $T$. dicoccum and T. monococcum, followed by 0.038 between 2 pairs i.e. Secale cereal EC 481695 and UP 2554; Aegilops squarrosa and Triticum dicoccum, followed by 0.039 between 2 pairs i.e. Secale cereal EC 481697 and UP 2425; Secale cereale EC 481695 and T. monococcum.

\section{References}

Ali, S., Azfer, M. A., Bashamboo, A., Mathur, P. K. and Malik, P. K. 1999. Characterization of species specific repetitive DNA from a highly endangered wild animal Rhinoceros unicornis and assessment of genetic polymorphism by microsatellite 
associated sequence amplification (MASA). Gene, 228: 33-42.

Anon 1996. Romanovs find closure in DNA. Nat. Genet.12: 339-340.

Bahieldin, A., Mahfouz, H. T., Eissa, H. F. et $a l ., 2005$. Field evaluation of transgenic wheat plants stably expressing the HVA1 gene for drought tolerance. Physiologia Plantarum123(4): $421-$ 427.

Bougot, Y., Lemoine, J., Pavoine, M. T., Barloy, D. and Doussinault, G. 2002. Identification of a microsatellite marker associated with $\mathrm{Pm} 3$ resistance alleles to powdery mildew in wheat. Plant Breeding 121(4): 325-329.

Dib, C., Faure, S., Fizames, C., Samson, D. and Drouot, N. 1996. A comprehensive map of the human genome based on 5264 microsatellites. Nature380: 152154.

Dograr, N., Akin-Yalin, S. and Akkaya, M. S. 2000. Discriminating durum wheat cultivars using highly polymorphic simple sequence repeat DNA markers. Plant Breeding119 (4): 360-362.

Dong, P., Wei, Y. M., Chen, G. Y. et al., 2009. EST-SSR diversity correlated with ecological and genetic factors of wild emmer wheat in Israel. Hereditas, 146(1): 1-10.

Dreisigacker, S., Melchinger, A. E., Zhang, P., Ammar, K., Flachenecker, C., Hoisington, D. and Warburton, M. L. 2000. Hybrid performance and heterosis in spring bread wheat, and their relations to SSR-based genetic distances and coefficients of parentage. Euphytica144: 51-59.

Driesigacker, S., Zhang, P., Ginkel, M. V., Warburton, M., Hoisington, D., Bohn, M. and Melchinger, A. E. 2004. SSR and pedigree analyses of genetic diversity among CIMMYT wheat lines targeted to different megaenvironments. Crop Sci., 44: 381-388.
El Maghraby, M. A., Moussa, M. E., Hana, N. S., Agrama, H. A. 2005. Combining ability under drought stress relative to SSR diversity in common wheat. Euphytica 141: 301 - 308.

Fahima, T., Röder, M. S., Wendehake, K., Kirzhner, V. M. and Nevo, E. 2002. Microsatellite polymorphism in natural populations of wild emmer wheat, Triticum dicoccoides in Israel. Theoretical and Applied Genetics104(1):17-29.

FAOSTAT faostat.fao.org/www.faostat.fao. org.

Fleury, D., Jefferies, S., Kuchel, H. and Langridge, P. 2010. Genetic and genomic tools to improve drought tolerance in wheat. Journal of Experimental Botany61(12): 32113222.

Gao, L. F., Jing, R. L., Huo, N. X., Li, Y., Li, X. P., Zhou, R. H., Chang, X. P., Tang, J. F., Ma, Z. Y. and Jia, J. Z. 2004. One hundred and one new microsatellite loci derived from ESTs (EST-SSRs) in bread wheat. Theor. Appl. Genet. 108: 1392-1400.

Habash, D. Z., Kehel, Z., and Nachit, M. 2009. Genomic approaches for designing durum wheat ready for climate change with a focus on drought. Journal of Experimental Botany60(10): 2805-2815.

Hayden, M. J., Stephenson, P., Logojan, A. M., Khatkar, D., Rogers, C., Elsden, J., Koebner, R. M.D., Snape, J. W. and Sharp, P. J. 2006. Development and genetic mapping of sequence-tagged microsatellites (STMs) in bread wheat (Triticum aestivum L.). Theor. Appl. Genet. 113: 1271-1281.

Hoisington, D., Bohorova, N., Fennell, S., Khairallah, M., Pellegrineschi, A., Ribaut, J. M. 2002. The application of biotechnology to wheat improvement. FAO corporate document repository: pp 
567.

Kantar, M., Lucas, S. J. and Budak, H. 2011. Drought stress: molecular genetics and genomics approaches. Advances in Botanical Research57: 445-493.

Kantar, M., Lucas, S. J. and Budak, H. 2011. miRNA expression patterns of Triticum dicoccoides in response to shock drought stress. Planta, 233(3): 471-484.

Krugman, T., Chagué, V., Peleg Z. et al., 2010. Multilevel regulation and signalling processes associated with adaptation to terminal drought in wild emmer wheat. Functional and Integrative Genomics, 10(2): 167-186.

Kuleung, C., Baenziger, P. S. and Dweikat, I. 2004. Transferability of SSR markers among wheat, rye and triticale. Theor. Appl. Genet.108 (6): 1147-1150.

Kumar, U., Joshi, A. K., Kumari, M., Paliwal, R., Kumar, S. and Roder, M. S. 2010. Identification of QTLs for stay green trait in wheat (Triticum aestivum L.) in the "Chirya 3" $\times$ "Sonalika" population. Euphytica 174 (3): 437-445.

Litt, M. and Lutty, J. A. 1989. A hypervariable microsatellite revealed by in vitro amplification of a dinucleotide repeat within the cardiac muscle actin gene. Am. J. Hum. Genet. 44: 397-401.

Lucas, S. Dogan, E. and Budak, H. 2011. TMPIT1 from wild emmer wheat: first characterisation of a stress-inducible integral membrane protein. Gene483, (1-2): 22-28.

Maccaferri, M., Sanguineti, M. C., Corneti, S. et al., 2008. Quantitative trait loci for grain yield and adaptation of durum wheat (Triticum durum Desf.) across a wide range of water availability. Genetics 178(1): 489-511.

Mathews, K. L. Malosetti, M. Chapman, S. et al., 2008. Multi-environment QTL mixed models for drought stress adaptation in wheat. Theoretical and Applied Genetics, 117(7): 1077-1091.
Medini, M., Hamza, S., Rebai, A. and Baum, M. 2005. Analysis of genetic diversity in Tunisian durum wheat cultivars and related wild species by SSR and AFLP markers. Genetic Resources and Crop Evolution, 52(1): 21 - 31.

Meena, R., Tripathi, S., Chander, S., Chookar, R., Verma, M., and Sharma, R. 2015. Identifying drought tolerant wheat varieties using different indices. SAARC Journal of Agriculture, 13(1), 148-161.

Mir R. R., Kumar J., Balyan H. S. and Gupta P. K. 2012. A study of genetic diversity among Indian bread wheat (Triticum aestivum L.) cultivars released during last 100 years. Genet. Resour. Crop Evol., 59: 717-726.

Nagarajan S. 2005. Can India produce enough wheat even by 2020? Curr. Sci., 89: 1467-1471.

Nevo, E. and Chen, G. 2010. Drought and salt tolerances in wild relatives for wheat and barley improvement. Plant, Cell \& Environment33(4): 670-685.

Pajayl, M., Sorrells, M. E., Baum, M., Wolters, P. and Powell, W. 2002. Isolation of EST-derived microsatellite markers for genotyping the A and B genomes of wheat. Theor. Appl. Genet. 104: 399-407.

Peleg, Z., Fahima, T., Abbo, S. et al., 2005. Genetic diversity for drought resistance in wild emmer wheat and its ecogeographical associations. Plant, Cell \&Environment, 28(2): 176-191.

Peng, J. H., Sun, D. F., Peng, Y. L. and Nevo, E. 2013. Gene discovery in Triticum dicoccoides, the direct progenitor of cultivated wheats. Cereal Research Communications, 41(1): 1-22.

Roca, A. L., Georgiadis, N., Peacon, S. J. and O'Brien, S. J. 2001. Genetic evidence for two species of elephant in Africa. Science, 293: 1473-1477.

Rubinsztein, C. D., Amos, W., Leggo, J., Goodburn, S. and Jain, S. 1995. 
Microsatellite evolution-evidence for directionality and variation in rate between species. Nat. Genet.10: 337343.

Sharma, S., Balyan, H. S., Kulwal, P. L., Kumar, N., Varshney, R. K., Prasad, M. and Gupta, P. K. 2002. Study of interspecific SSR polymorphism among 14 species from Triticum-aegilops group. Wheat Information Service 95: 23-28.

Sio-Se Mardeh, A., Ahmadi, A. Poustini, K. and Mohammadi, V. 2006. Evaluation of drought resistance indices under various environmental conditions. Field Crop Research98: 222-229.

Song, Q. J., Shi, J. R., Singh, S., Fickus, E. W., Costa, J. M., Lewis, J., Gill, B. S., Ward, R. and Cregan, P. B. 2005. Development and mapping of microsatellite (SSR) markers in wheat. Theor. Appl. Genet. 100: 550-560.

Torada, A., Koike, M., Mochida, K. and Ogihara, Y. 2006. SSR-based linkage map with new markers using an intraspecific population of common wheat. Theor. Appl. Genet. 112: 10421051.

\section{How to cite this article:}

Payal Saxena, Usha Pant and Khanna, V. K. 2020. The Potential of SSR Markers to Reveal the Genetic Diversity among Wheat and its Wild Relatives and to Test the Hybridity of $F_{1} s$. Int.J.Curr.Microbiol.App.Sci. 9(05): 2686-2699. doi: https://doi.org/10.20546/ijcmas.2020.905.308 